\title{
Alterations in Memory Networks in Mild Cognitive Impairment and Alzheimer's Disease: An Independent Component Analysis
}

\author{
Kim A. Celone, ${ }^{1,2}$ Vince D. Calhoun, ${ }^{3,4}$ Bradford C. Dickerson, ${ }^{1,2}$ Alireza Atri, ${ }^{2}$ Elizabeth F. Chua, ${ }^{1}$ Saul L. Miller, ${ }^{1,2}$ \\ Kristina DePeau, ${ }^{1,2}$ Doreen M. Rentz, ${ }^{1}$ Dennis J. Selkoe, ${ }^{1}$ Deborah Blacker, ${ }^{1,2}$ Marilyn S. Albert, ${ }^{2,5}$ and Reisa A. Sperling ${ }^{1,2}$ \\ ${ }^{1}$ Memory Disorders Unit, Department of Neurology, Brigham and Women's Hospital, Harvard Medical School, Boston, Massachusetts 02115, ${ }^{2}$ Departments \\ of Neurology, Psychiatry, and Radiology, and Athinoula A. Martinos Center for Biomedical Imaging, Massachusetts General Hospital, Boston, \\ Massachusetts 02114, ${ }^{3}$ Olin Neuropsychiatry Research Center, The Institute of Living/Hartford Hospital, Hartford, Connecticut 06106, ${ }^{4}$ Department of \\ Psychiatry, Yale University School of Medicine, New Haven, Connecticut 06511, and 5Department of Neurology, Johns Hopkins University School of \\ Medicine, Baltimore, Maryland 21205
}

Memory function is likely subserved by multiple distributed neural networks, which are disrupted by the pathophysiological process of Alzheimer's disease (AD). In this study, we used multivariate analytic techniques to investigate memory-related functional magnetic resonance imaging (fMRI) activity in 52 individuals across the continuum of normal aging, mild cognitive impairment (MCI), and mild AD. Independent component analyses revealed specific memory-related networks that activated or deactivated during an associative memory paradigm. Across all subjects, hippocampal activation and parietal deactivation demonstrated a strong reciprocal relationship. Furthermore, we found evidence of a nonlinear trajectory of fMRI activation across the continuum of impairment. Less impaired MCI subjects showed paradoxical hyperactivation in the hippocampus compared with controls, whereas more impaired MCI subjects demonstrated significant hypoactivation, similar to the levels observed in the mild AD subjects. We found a remarkably parallel curve in the pattern of memory-related deactivation in medial and lateral parietal regions with greater deactivation in less-impaired MCI and loss of deactivation in more impaired MCI and mild AD subjects. Interestingly, the failure of deactivation in these regions was also associated with increased positive activity in a neocortical attentional network in $\mathrm{MCI}$ and $\mathrm{AD}$. Our findings suggest that loss of functional integrity of the hippocampal-based memory systems is directly related to alterations of neural activity in parietal regions seen over the course of $\mathrm{MCI}$ and $\mathrm{AD}$. These data may also provide functional evidence of the interaction between neocortical and medial temporal lobe pathology in early $\mathrm{AD}$.

Key words: Alzheimer's disease; mild cognitive impairment; fMRI; hippocampus; default mode network; independent component analyses

\section{Introduction}

Memory impairment, in particular the loss of the ability to form and retain new episodic memories, is the hallmark of early Alzheimer's disease (AD). This impairment is often attributed to synaptic dysfunction and neuronal loss in the perforant pathway within the medial temporal lobe (MTL), serving to "disconnect"

Received Jan. 12, 2006; revised Aug. 4, 2006; accepted Aug. 27, 2006.

This work was supported by National Institute on Aging Grant P01-AG04953, National Institute of Neurological Disorders and Stroke Grant K23-NS02189, Massachusetts Aging and Disability Resource Consortium Grant P50AG05134, National Center for Research Resources Grant P41-RR14075, the Beeson Scholars in Aging Program (American Federation for Aging Research), Eli Lilly Pharmaceuticals, and the Harvard Center for Neurodegeneration and Repair. We thank the staff of the Massachusetts General Hospital Gerontology Research Unit and Brigham and Women's Hospital Memory Disorders Clinical Research Unit for assistance with subject recruitment, evaluation, and data management, Dr. Steven Salloway for assistance with Alzheimer's disease patient recruitment, and Mary Foley, Larry White, and the Martinos Center staff for assistance with magnetic resonance imaging data collection.

Correspondence should be addressed to Dr. Reisa A. Sperling, Memory Disorders Unit, Brigham and Women's Hospital, 221 Longwood Avenue, Boston, MA 02115. E-mail: reisa@rics.bwh.harvard.edu.

D0I:10.1523/JNEUROSCI.2250-06.2006

Copyright $\odot 2006$ Society for Neuroscience ～0270-6474/06/2610222-10\$15.00/0 the hippocampus from neocortical association areas (Hyman et al., 1984). Alterations in functional magnetic resonance imaging (fMRI) activation have been reported in mild cognitive impairment (MCI) and AD. The majority of these studies, including our own, have focused on activation within the MTL, and much less is known about activity in regions that are functionally connected to the MTL in MCI and AD. Several fMRI studies in MCI and AD have also reported alterations in "deactivation" (i.e., taskinduced reductions in MR signal, compared with rest or control conditions), particularly in the posterior cingulate, precuneus, and lateral parietal regions (Lustig et al., 2003; Greicius et al., 2004; Rombouts et al., 2005b). The relationship between alterations in memory-related activation within the MTL and failure of deactivation in these parietal regions over the course of MCI and $\mathrm{AD}$ remains to be elucidated.

In this study, we used independent component analyses (ICA) to investigate the pattern of fMRI activity during an associative memory task. Applied to fMRI, ICA extracts functional networks by identifying spatially independent and temporally synchronous 
regions of the brain without a priori knowledge of the paradigm. This analytic approach is particularly advantageous to examine the interaction between regions or "networks" that activate or deactivate during cognitive tasks. In addition, this data-driven technique is capable of decomposing the complex temporal MR signal patterns which comprise the overall blood oxygen leveldependent (BOLD) response, and may allow the discovery of additional temporal components that are not identified with traditional fMRI “subtraction” analyses.

We wanted to investigate patterns of memory-related fMRI activity across the continuum of normal aging to $\mathrm{AD}$, as the transitional phase characterized as MCI encompasses a heterogeneous group of older individuals. Relatively few MCI fMRI studies have been published to date, which have used a variety of criteria to define MCI. The patterns of fMRI MTL activation in MCI have also been quite variable, ranging from decreased activation at levels similar to $\mathrm{AD}$, to paradoxically increased activation above the level of controls (Small et al., 1999; Machulda et al., 2003; Dickerson et al., 2004, 2005; Johnson et al., 2004). Based on our previous work and reports from other groups studying atrisk subjects (Bookheimer et al., 2000; Bondi et al., 2005; Bassett et al., 2006) we hypothesize that there is a "nonlinear" trajectory of memory related $\mathrm{AMRI}$ activation over the course of prodromal $\mathrm{AD}$ that includes a phase of MTL "hyperactivation" in early stages, followed by decreased MTL activation in later stages of MCI and mild AD. Furthermore, we hypothesize that these alterations in MTL activation will be strongly related to the alterations in deactivation of neocortical networks over the course of MCI and $\mathrm{AD}$.

\section{Materials and Methods \\ Study participants}

Fifty-two older individuals participated in the study. All subjects provided informed consent in accordance with the Human Research Committee guidelines of the Massachusetts General Hospital and Brigham and Women's Hospital (Boston, MA).

Forty-one of the participants were recruited from a longitudinal study examining preclinical predictors of Alzheimer's disease. The remaining eleven subjects (MCI, $n=1$; $\mathrm{AD}, n=10$ ) were recruited from memory disorder clinics. A subset of these subjects ( $n=29$; normal older controls, 10 ; $\mathrm{MCI}, 9 ; \mathrm{AD}, 10)$ were studied previously in a report using anatomically defined regions of interest limited to the MTL (Dickerson et al., 2005).

Subjects were classified into four groups on the basis of their clinical dementia rating (CDR) scale, a standardized clinical assessment instrument (Morris et al., 1997). It is widely acknowledged that individuals with an overall rating of $\mathrm{CDR}=0.5$ is quite heterogeneous and likely represents a continuum between normal aging and mild AD that encompasses the spectrum of MCI (Petersen et al., 1997). Thus, we decided a priori to subdivide the CDR 0.5 subjects on the basis of their CDR sumof-box (CDR-SB) score. The CDR-SB measure further delineates the degree of functional impairment, and has been shown previously to correlate with the likelihood of conversion to clinical AD (Daly et al., 2000). Based on these previous data, we classified the CDR 0.5 subjects into two groups: low sum of box score (low-SB) MCI and high sum of box score (high-SB) MCI. The low-SB MCI $(n=15)$ had a total CDR rating of 0.5 , with at least a 0.5 in the memory box, and a CDR-SB score of 0.5-1.5. high-SB MCI $(n=12)$ had a total CDR rating of 0.5 , with at least a 0.5 in the memory box, and a CDR-SB of 2.0-3.5. We did not require that either group of MCI subjects perform below specific cutoffs on any objective psychometric tests. See Table 1 for subject demographics and neuropsychological test results.

Normal older control subjects $(\mathrm{NC} ; n=15)$ had a $\mathrm{CDR}=0.0$, and were followed longitudinally for at least one year with no evidence of cognitive decline. The patients with Alzheimer's disease $(n=10)$ met criteria for probable AD (McKhann et al., 1984) and had mild dementia
Table 1. Summary of subject demographic information

\begin{tabular}{lcccc}
\hline & NC & Low-SB MCl & High-SB MCl & AD \\
\hline$n$ & 15 & 15 & 12 & 10 \\
Age (years) & $75.5 \pm 6.0$ & $75.1 \pm 7.1$ & $80.0 \pm 4.5^{*}$ & $77.6 \pm 8.0$ \\
Education (years) & $16.5 \pm 2.1$ & $17.1 \pm 2.6$ & $15.3 \pm 3.7$ & $12.5 \pm 2.8^{*}$ \\
Sex (male/female) & $7 / 8$ & $8 / 7$ & $6 / 6$ & $3 / 7$ \\
CDR & 0 & 0.5 & 0.5 & 1.0 \\
CDR-Sum of Boxes & 0 & $1.1 \pm 0.4$ & $2.5 \pm 0.6$ & $5.2 \pm 0.9$ \\
CVLT (total learning) & $55.6 \pm 6.7^{*}$ & $42.4 \pm 6.6$ & $46.1 \pm 14.6$ & na \\
CVLT (delayed recall) & $12.9 \pm 2.9$ & $9.5 \pm 2.1$ & $8.3 \pm 4.7$ & na \\
MMSE & $29.5 \pm 0.5$ & $29.3 \pm 0.9$ & $28.6 \pm 1.2$ & $21.1 \pm 3.2^{*}$ \\
\hline
\end{tabular}

${ }^{*} p<0.05$ difference from other groups. Data are presented as mean \pm SD. CVLT, California Verbal Learning Test; na, not applicable.

severity as characterized by an overall CDR of 1.0 [Mini-Mental State Exam (MMSE): mean, 21.1; range, 15-24]. All AD patients had either been off cholinesterase inhibitors for at least $30 \mathrm{~d}$ before scanning or had never taken these medications.

\section{MRI procedures}

Data acquisition. Subjects were scanned using a Siemens (Iselin, NJ) Trio 3.0 tesla scanner with a three-axis gradient head coil. Functional echo planar image data were acquired using a $\mathrm{T} 2{ }^{\star}$-weighted gradient echo planar imaging (EPI) pulse sequence (repeat time/echo time, 2500/30 $\mathrm{ms}$; flip angle, $90^{\circ}$ ). Twenty-eight slices were acquired in an oblique coronal orientation beginning at the occipital pole, perpendicular to the anterior-posterior commissure line, to maximize in-plane resolution, and minimize susceptibility artifact in the hippocampus $(5 \mathrm{~mm}$ thick with $1 \mathrm{~mm}$ gap; voxel dimensions, $3.125 \times 3.125 \times 6 \mathrm{~mm})$. Scanning time for each functional run was $4 \mathrm{~min}$ and $15 \mathrm{~s}$, consisting of 102 time points per run. Six functional runs were acquired for each subject.

fMRI memory paradigm. The associative memory task consisted of novel faces (faces unfamiliar to the participants) paired with fictional first names (Sperling et al., 2001, 2002, 2003b). Participants were explicitly instructed to try to remember the name associated with each face, and to make a decision regarding whether they thought the name "fit" the face (i.e., "was a good name for that face") or not. This is a purely subjective decision, which has been shown to enhance associative encoding (Sperling et al., 2003a). The stimuli were arranged in a block design and each run consisted of three conditions: novel face-name pairs, repeated face-name pairs, and fixation. During the 12 novel blocks, the face-name pairs were presented once for $5 \mathrm{~s}$. Subjects viewed seven novel face-name pairs during each novel block, for a total of 84 novel face-name pairs over the course of the session. The 12 repeated blocks consisted of one male and one female alternating face-name pair presented for $5 \mathrm{~s}$ each, and were introduced to the subject immediately before the functional scan in a practice run. Each repeated face-name pair was presented a total of 42 times over the course of the session. Lastly, during a fixation condition, a white cross was presented in between face-name pairs and blocks. There were three blocks of fixation between the novel and repeated blocks, each lasting $25 \mathrm{~s}$, as well as $5 \mathrm{~s}$ of fixation at the beginning and end of each run. The participants were instructed to look at the fixation cross to focus the subject's visual attention.

Postscan memory task. Approximately $5 \mathrm{~min}$ after the scanning session concluded, all subjects completed two brief memory tests, presented on a computer screen outside of the scanner, consisting of a face-recognition test and forced-choice associative recognition test. The first memory test (MT1) consisted of 12 of the novel faces presented only once during the experiment, the two repeated faces, as well as eight faces not seen during the experiment that were presented as foils. Subjects were instructed to indicate whether they had seen the face while in the scanner or not. The second memory test (MT2) was a two-forced-choice associative recognition task during which a different set of 12 novel faces seen during the experiment as well as the two repeated faces were presented. Each face was shown with two names printed underneath: the correct name that was paired with the face during scanning and one incorrect name that was previously paired with a different face during scanning. The position of the correct name was counterbalanced across the postscan test stimuli, 
and subjects were instructed to indicate the correct name by pointing to it on the computer monitor. Note that a forced-choice recognition task was used so that $\mathrm{AD}$ subjects could perform above chance levels, as it is relatively less difficult than delayed recall measures typically used in evaluation of less impaired subjects.

\section{Data analysis}

Functional MRI data were preprocessed using statistical parametric mapping (SPM2; Wellcome Department of Cognitive Neurology) for Matlab (MathWorks, Natick, MA). Functional images runs were realigned using INRIAlign, a motion correction algorithm unbiased by local signal changes. The data were then normalized to the standard SPM2 EPI template and resliced into $3 \times 3 \times 3 \mathrm{~mm}$ resolution in Montreal Neurological Institute (MNI) space. The registration of the EPI data to the template was checked for each individual subject. The fMRI data were smoothed with a Gaussian kernel of $8 \mathrm{~mm}$. The sequence of temporal events were modeled with an SPM2 design matrix including a regressor, convolved with the canonical hemodynamic response function, and a discrete cosine transform implementing a high-pass filter of $260 \mathrm{~s}$ was used to filter out low-frequency variations (i.e., drift across each run). A high-pass filter of $260 \mathrm{~s}$ was chosen on the basis that independent component analysis does not extract components based on blocks of data, but across an entire run, therefore we were interested in the long term temporal dynamics of the data. Also, one advantage of using a technique such as ICA, is that it is capable of extracting noise, whether caused by motion, low frequency drift, or other common physiologic artifacts. Therefore, at the initial preprocessing level, we did not impose a traditional high-pass filter so as not to interfere with the ICA's detection of low-frequency noise.

The independent component analysis was performed using a group ICA for fMRI toolbox [GIFT; icatb.sourceforge.net (Correa et al., 2005)]. The toolbox uses a group approach involving an initial ICA estimation on concatenated data, followed by the computation of subject-specific components and time courses. This subject-wise concatenation approach has been shown to be a useful approach to group ICA analysis (Calhoun et al., 2001; Schmithorst and Holland, 2004). The toolbox performs the analysis in three stages: (1) data reduction, (2) application of the ICA algorithm, and (3) back reconstruction. The data reduction step uses principal component analysis to reduce the individual subjects' data in dimension. These data are then further reduced, resulting in a matrix that can be used during the back reconstruction stage. In the second stage, the estimation of independent sources is performed using the Infomax algorithm (Bell and Sejnowski, 1995). It is in the course of this stage that spatially independent functional maps are created. The final stage, back reconstruction, consists of computing individual subject image maps and time courses, followed by the process of grouping components across subjects and thresholding the resulting group ICA images (Calhoun et al., 2001).

Twenty independent components (ICs) were extracted from each group's fMRI dataset. The GIFT dimensionality estimation tool was used to determine the number of components that would accurately represent the data. Each IC consists of a spatial map and a temporal profile. The spatial maps contain voxels that are positively and negatively correlated with the IC's time course. Both the spatial pattern and frequency spectra of each component were visually inspected to determine its relationship to task and possible physiological artifacts (e.g., external head movement, eye movements, and CSF pulsation within ventricles). The components were then sorted on the basis of the multiple regression of each component's time course with the timing of the stimulus paradigm, including novel, repeated, and fixation conditions, assessed by examining the $R^{2}$ value. ICs of interest were generated by first averaging within subject, across all six runs, to create individual subject component maps. Positively and negatively correlated voxels were examined separately for each IC. The individual subject data were then entered into SPM2 randomeffects analyses one- and two-sample $t$ tests. The one-sample $t$ tests were masked with a within-group mask of component activity of interest thresholded at $p<0.01$. At the two-sample $t$ test level the pairwise group differences were masked with a union mask of the component activity of interest from the two groups being compared (also thresholded at $p<$

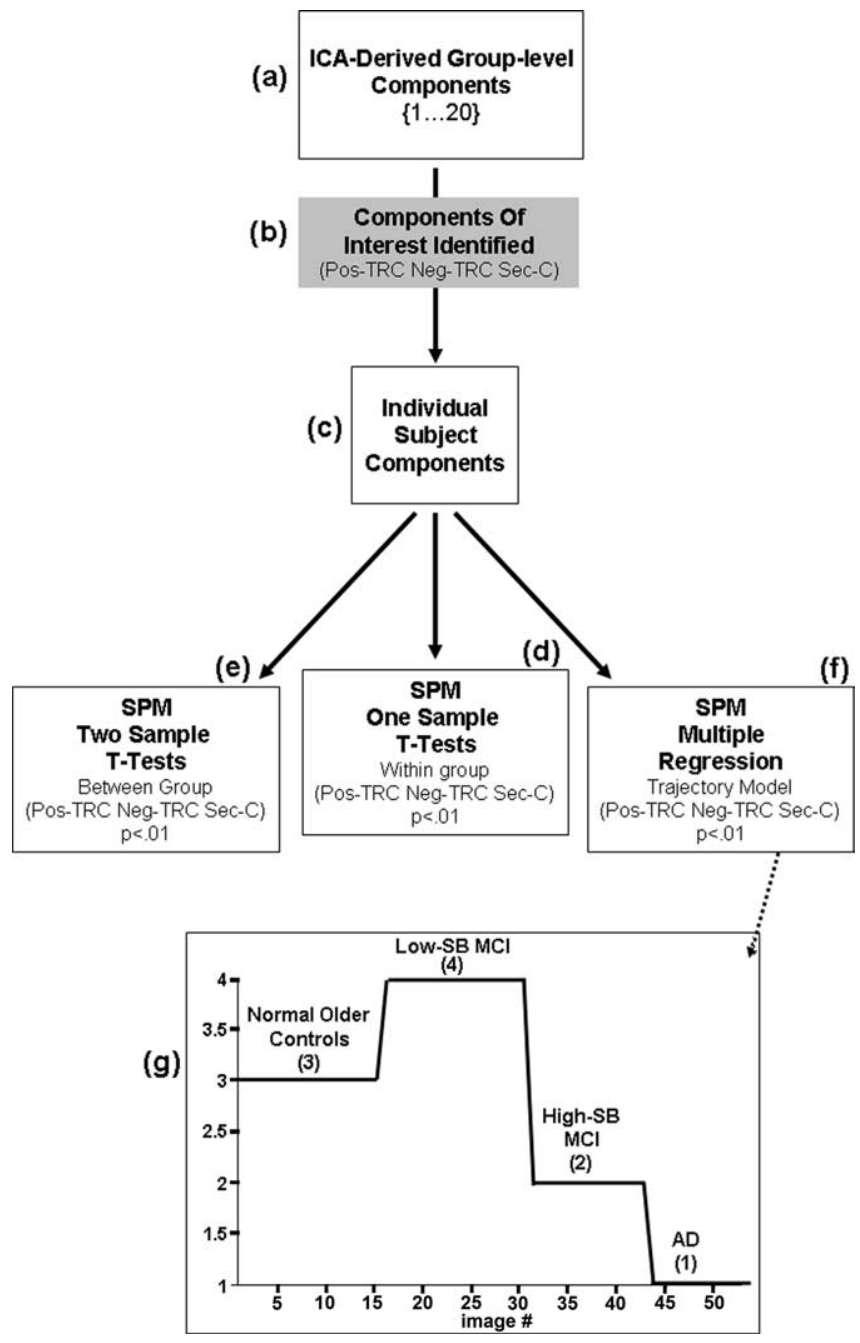

Figure 1. Analysis methods diagram. Visual representation of the analysis methods are depicted in a flow chart. $\boldsymbol{a}$, Starting at the top, 20 ICs were extracted from each group's fMRI dataset. $\boldsymbol{b}$, The components were then sorted on the basis of the multiple regression of each component's time course with the timing of stimulus paradigm. c, ICs of interest were generated by first averaging within subject, across all six runs, to create individual subject component maps. $\boldsymbol{d}$ - $\boldsymbol{f}$, The individual subject data were then entered into SPM2 random-effects analyses one-sample ( $\boldsymbol{d}$ ) and two-sample (e) $t$ tests, as well as multiple regression models which tested the nonlinear relationship across all four groups $(\boldsymbol{f}) . \boldsymbol{g}$, The SPM design matrix visually represents the model that was tested across all four subject groups for each IC of interest. The design matrix is labeled by group and covariate $[3, \mathrm{NC} ; 4$, low-SB MCl; 2, high-SB MCl; $1, A D]$ that corresponds to each group.

0.01). A diagram of the methods has been provided in Figure $1 a$ to further clarify the sequence of the analytic processes (for a full listing of all abbreviations, see the glossary located in the supplemental material, available at www.jneurosci.org).

Previous research has suggested that subjects at risk for $\mathrm{AD}$, on the basis of genetic risk, family history or in the very mild stages of MCI, may show a paradoxical increase in task-related activity (Bookheimer et al., 2000; Dickerson et al., 2005; Bassett et al., 2006), whereas studies of more impaired MCI subjects have reported a decrease in task-related fMRI activity (Machulda et al., 2003; Johnson et al., 2004). To test the hypothesis of a nonlinear trajectory over the continuum of aging to mild AD, multiple regression models were constructed in SPM for each IC of interest across all subjects. A single covariate was used to describe the hypothesized level of activity for each group, more specifically the model tested whether voxel activity displayed a pattern of increased activity above normal controls in the early phases of the disease process (low-SB $\mathrm{MCI}$ ), followed by a decrease in activity just before clinical onset of $\mathrm{AD}$ 


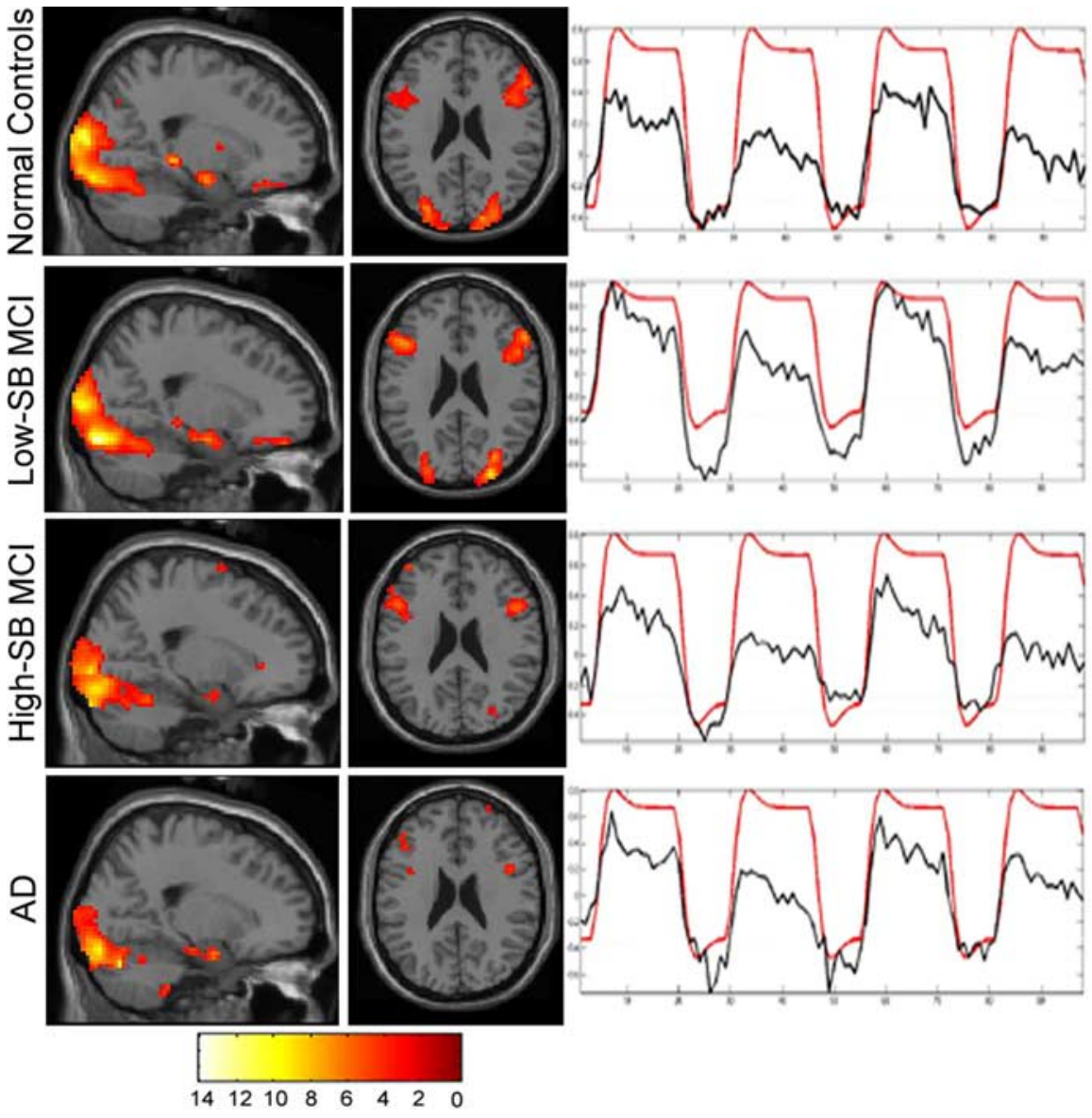

Figure 2. ICA-derived Pos-TRC. Sagittal and axial slices, display the spatial pattern of Pos-TRC activity across groups ( $p<$ 0.01). Sagittal and axial slices are overlaid on the SPM2 single subject T1 template. The left hemisphere is displayed on the left. The colorscale represents the resultant $\mathrm{T}$ scores from testing whether the component's voxels indicate percent signal change similarly to the deviations present in the estimated time course. Neuroanatomic regions involved in this component include the hippocampus, bilateral inferior frontal, fusiform, and visual association cortices. Time courses represent the temporal profile of each component across group (black) overlaid on the paradigm "box-car" design (red), demonstrating the degree of task-relatedness of the Pos-TRC component [NC, $R^{2}=0.866$; low-SBMCl, $R^{2}=0.915$; high-SB MCl, $R^{2}=0.870 ; A D, R^{2}=0.891(p<$ $0.000001)]$

(high-SB MCI), where voxel activity is intermediate to normal controls and that of $\mathrm{AD}$ patients. The covariate matrix entered into the model was as follows: $\mathrm{NC}=3$; low-SB MCI $=4$; high-SB MCI $=2 ; \mathrm{AD}=1$ (Fig. $1 b$ ).

To explore the extent of activation or deactivation in specific brain regions involved in the ICs of interest, a region of interest (ROI) analysis was also implemented. Each region that was identified as contributing significantly to the IC was matched to a standardized ROI available within the Marsbar library of ROIs (Brett et al., 2002). The extent variables were then calculated from a count of voxels above a threshold of $p<$ 0.01 within each ROI mask for each individual subject.

\section{Results}

ICA findings: task-related component: within-group analyses Each of the four subject groups generated a single task related component that was highly correlated with task (temporally associated with the timing of the block design paradigm for both novel and repeated conditions (NC, $R^{2}=0.866$; low-SB MCI, $R^{2}=0.915$; high-SB MCI, $R^{2}=0.870 ; \mathrm{AD}, R^{2}=0.891 ; p<$ $0.000001)$. This component included voxels that were directly (positively) correlated with the component time course [positive task-related component (Pos-TRC)], and voxels inversely (negatively) correlated with the component time course [negative task-related component (Neg-TRC)].
The Pos-TRC represents voxels that demonstrated an increase in MR signal during the encoding of face-name pairs (novel and repeated blocks). The Pos-TRC revealed a very consistent overall spatial and temporal pattern of activity across all groups (Fig. 2). The Pos-TRC included the hippocampal formation, inferior prefrontal, fusiform, and surrounding visual association cortices bilaterally in all groups. The NC and low-SB MCI groups showed significant activity in the Pos-TRC along the longitudinal axis of the hippocampus (normal control peaks [all peak magnitudes and cluster sizes $(k)$ are reported at $p<0.0001$ unless otherwise stated]: anterior hippocampus $[Z=4.3 ; x=24, y=$ $-9, z=-1 ; k=41]$, posterior hippocampus $[Z=4.7 ; x=24, y=-30, z=-3 ; k=$ 53]; low-SB MCI peaks: anterior hippocampus $[Z=4.5 ; x=24, y=-9, z=$ $-18 ; k=63]$, posterior hippocampus $[Z=4.6 ; x=-21$, $y=-30, z=-9 ; k=136])$, whereas the high-SB MCI and AD groups showed small amounts of activity only at the very anterior hippocampus/amygdala border [high-SB MCI peak $(Z=4.69 ; x=21, y=$ $0, z=-18 ; k=15) ; \operatorname{AD}$ peak $(Z=3.7 ; x=$ $24, y=-3, z=-21 ; k=6)]$.

The Neg-TRC represented voxels demonstrating a decrease in MR signal during the encoding of face-name pairs (or relatively greater MR signal during the control condition of visual fixation). The Neg-TRC also showed an overall similar spatial-temporal pattern of activity within each group, including the precuneus, posterior cingulate, lateral parietal and temporal cortices bilaterally, as well as the anterior cingulate and medial frontal regions (Fig. 3). This set of regions has been described previously as being involved in the "default-mode of brain activity" (Raichle et al., 2001; Greicius et al., 2003). The Low-SB MCI group in particular, showed a large extent of Neg-TRC activity $(k=867)$ in the precuneus $(Z=5.3 ; x=6, y=-60, z=39)$ and posterior cingulate $(Z=$ $5.6 ; x=15, y=-42, z=27)$.

\section{Relationship between activation and deactivation within the task-related component}

Across all subjects, we found clear evidence of an inverse relationship between the extent of activity in the Pos-TRC and the Neg-TRC across all of the groups (Fig. 4). Specifically, the extent of Pos-TRC activity within the hippocampal ROI was highly correlated with the extent of Neg-TRC activity within the precuneus $(r=0.775 ; p<0.0001)$, bilateral parietal regions $(r=0.896 ; p<0.0001)$, and the posterior cingulate $(r=$ $0.568 ; p<0.0001)$ across all subjects. These findings support the hypothesis of a reciprocal relationship between the extent of activation in the hippocampus and extent of deactivation within parietal regions comprising the default network. 
Multiple regression model within the task-related component

The results of testing the regression model for evidence of a nonlinear trajectory across all four subject groups (low-SB MCI $>$ normal controls $>$ high-SB $\mathrm{MCI}>\mathrm{AD}$ ) yielded similar results to between group $t$ test results from above. This model was significant at $p<0.001$ in multiple regions, and provides additional evidence of curvilinear trajectory over the course of MCI. Regions with the strongest relationship to the regression model for the Pos-TRC are shown in Figure $5 a$ and include hippocampal formation bilaterally (left hemisphere, $Z=5.46$; right hemisphere, $Z=5.51$ ), inferior frontal, and cingulate gyri, as well as the dorsolateral prefrontal cortex and visual association cortices (Table 2). Testing the identical regression model on the Neg-TRC activity across all four subject groups also suggests that the regions involved in the negatively correlated activity within the task-related component may be affected similarly to the positively correlated task-related activity. Regions with the strongest relationship to the regression model for the Neg-TRC include the posterior and anterior cingulate, precuneus $(Z=6.72 ; x=3, y=-60$, $z=54$ ), and lateral parietal cortex (Fig. 5b, Table 2).

Between-group comparisons for the task-related component

We examined between-group differences for both the Pos-TRC and Neg-TRC using SPM2 whole-brain and ROI analyses. The low-SB MCI subjects demonstrated increased expression of the Pos-TRC compared with NC $(p<$ $0.0001)$ in the bilateral hippocampus $(Z=4.9$, left hippocampus MNI coordinates, $x=-18, y=-18, z=-15, k=116$; right hippocampus, $Z=4.7 ; x=27, y=-15, z=-15 ; k=15)$ and bilateral inferior frontal $(Z=5.2 ; x=-48, y=21, z=21$; $k=126$; and $Z=5.5 ; x=57, y=24, z=18 ; k=44$, respectively) (Fig. 5a). High-SB MCI showed significantly decreased activation compared with NC, particularly in the hippocampus (Fig. $5 c$ ), supporting the hypothesis of a nonlinear trajectory of MTL activation over the course of MCI. The NC and the low-SB MCI subjects exhibited similar expression of fusiform activation, which was significantly greater than both the high-SB MCI and AD groups $(p<0.001)$. ROI analyses also revealed significant differences across the groups in the extent of active voxels within the MNI-defined ROIs for bilateral hippocampal formation, the bilateral inferior frontal (including opercular, orbital, and triangular regions), and bilateral fusiform cortices respectively (all peaks reported at $p<0.0001$ unless otherwise stated) (Figs. $5 c, d, 6)$.

Significant between-group differences were also observed within the Neg-TRC. Low-SB MCI subjects demonstrated significantly greater expression of the Neg-TRC within the precuneus, posterior cingulate, and bilateral parietal regions in comparison to NC, as well as the high-SB MCI and AD subjects [low-SB

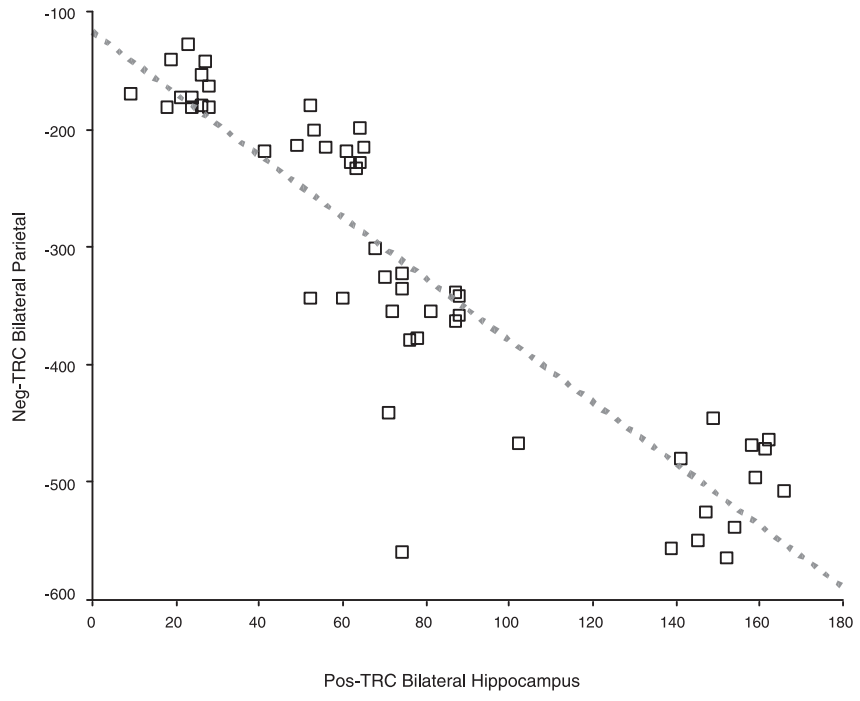

Figure 4. Relationship between activation and deactivation within the task-related component. Scatterplot illustrates the association of Pos-TRC bilateral hippocampal activity with the extent of Neg-TRC bilateral parietal activity (Pearson's $r=-0.896 ; p<0.0001$ ). The red dotted line represents the linear fit of this data. Note that the extent of deactivation is represented as a negative number, connoting a decrease in MR activity during task. 

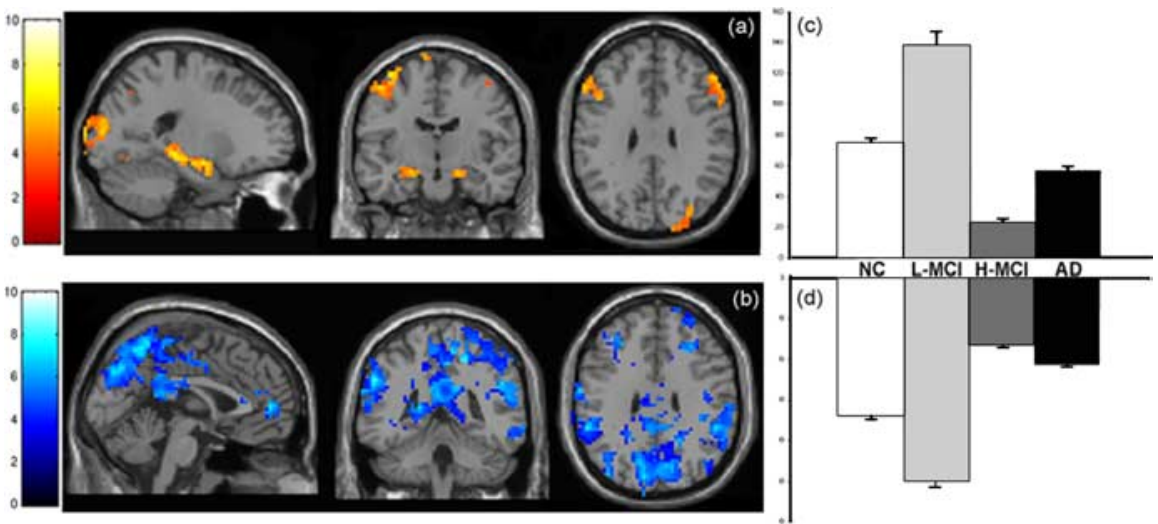

Figure 5. Nonlinear trajectory within the task-related component. Sagittal, coronal, and axial slices through the brain display the regions with the strongest positive relationship to the multiple regression model tested $(p<0.001)$. $\boldsymbol{a}$, Within the Pos-TRC, these regions include the left and right hippocampal formation, left and right inferior frontal gyrus, left cingulate gyrus, as well as the right dorsolateral prefrontal cortex. $\boldsymbol{b}$, Within the Neg-TRC, the regions with the strongest positive relationship to the multiple regression model include the left anterior and posterior cingulate, the precuneus, as well as right and left lateral parietal cortex. (Note that the same regression model is tested in both $\boldsymbol{a}$ and $\boldsymbol{b}$, and the colormap difference is a function of relationship of the regions to temporal time course of the paradigm.) $\boldsymbol{c}, \boldsymbol{d}$, Bar graphs display the nonlinear trajectory observed across groups as measured by voxel counts (error bars indicate SE) within the bilateral hippocampus within the Pos-TRC as well as the bilateral parietal regions within the Neg-TRC. Both ROl showed significant differences between all groups $(p<0.0001)$.

Table 2. Summary of multiple regression peaks testing the model of a nonlinear trajectory of fMRI activation across the continuum of impairment

\begin{tabular}{|c|c|c|c|c|}
\hline \multirow[b]{2}{*}{ Region } & \multicolumn{3}{|l|}{ Peak } & \multirow[b]{2}{*}{$Z$ value } \\
\hline & $x$ & $y$ & $z$ & \\
\hline \multicolumn{5}{|l|}{ Pos-TRC } \\
\hline LH, Hippocampus & -21 & -30 & -9 & 5.46 \\
\hline RH, Hippocampus & 24 & -6 & -18 & 5.51 \\
\hline LH, Inferior frontal gyrus & -36 & 27 & -6 & 5.60 \\
\hline RH, Inferior frontal gyrus & 39 & 27 & -6 & 4.31 \\
\hline $\mathrm{LH}$, Cingulate gyrus & -6 & 15 & 42 & 4.92 \\
\hline $\mathrm{RH}$, Dorsolateral prefrontal cortex & 54 & 24 & 24 & 5.75 \\
\hline \multicolumn{5}{|l|}{ Neg-TRC } \\
\hline LH, Posterior cingulate & -3 & -42 & 21 & 5.41 \\
\hline LH, Anterior cingulate & -3 & 54 & 3 & 6.34 \\
\hline RH, Precuneus & 3 & -60 & 54 & 6.72 \\
\hline LH, Lateral parietal cortex & -54 & -45 & 33 & 6.48 \\
\hline RH, Lateral parietal cortex & 51 & -51 & 12 & 5.77 \\
\hline \multicolumn{5}{|l|}{ Sec-C (Positive activity) } \\
\hline LH, Inferior frontal gyrus & -39 & 21 & 9 & 5.41 \\
\hline LH, Posterior cingulate & -3 & -24 & 33 & 4.95 \\
\hline LH, Dorsal anterior cingulate & -3 & 18 & 42 & 6.45 \\
\hline
\end{tabular}

SPM model: $(\mathrm{NC}=3<$ low-SB MCI $=4 \gg$ high-SB MCI $=2>A D=1)$. LH, Left hemisphere; RH, right hemisphere.

MCI > NC: precuneus $(Z=6.9 ; x=5, y=-45, z=27)$; posterior cingulate $(Z=7.2 ; x=6, y=-60, z=39$; combined cluster $k=546)$; lateral parietal $(Z=6.2 ; x=51, y=-51, z=12$; $k=107$; and $Z=6.5 ; x=-39, y=-84, z=33 ; k=22$ )] (Fig. $5 b)$. In contrast, the high-SB MCI and AD groups exhibited decreased Neg-TRC activity within the posterior cingulate and bilateral parietal regions in comparison to the $\mathrm{NC}(p<0.0001)$ (Fig. $5 d$ ). These data suggest that the alterations in parietal deactivation mirrors the alterations in MTL activation over the course of prodromal AD.

The extent of activity within the frontal regions of the NegTRC revealed several interesting differences between groups. The low-SB MCI, high-SB MCI, and AD groups all displayed an increase in Neg-TRC activity in the anterior cingulate [low-SB MCI $(Z=6.2 ; x=-9, y=48, z=6 ; k=143)$; high-SB MCI $(Z=6.2$; $x=3, y=39, z=6 ; k=50) ; \mathrm{AD}(Z=4.6 ; x=0, y=33, z=18$; $k=18)$ ] compared with the NC. Interestingly, the AD subjects also exhibited an increase in expression of Neg-TRC activity within the superior frontal gyrus $(Z=$ 6.53; $x=-15, y=39, z=30 ; k=51$ ) compared with NC [all peak magnitudes and cluster sizes $(k)$ reported at $p<0.0001$ unless otherwise stated].

\section{Secondary component of interest: within-group analysis}

Visual inspection of the remaining nineteen components for each group revealed components that primarily represented artifact, both physiological (CSF pulsation) and external head motion. However, each group revealed one additional component showing predominantly positive activity in a strikingly similar pattern across the groups, and which did not appear to be artifactual by virtue of its spatial distribution and the frequency spectra (Marchini and Ripley, 2000; Moritz et al., 2003). Interestingly, this secondary component of interest (Sec-C) did not show a significant temporal relationship with the paradigm timing $\left(\mathrm{NC}, R^{2}=0.08\right.$; low-SB MCI, $R^{2}=0.02$; high-SB MCI, $R^{2}=0.13$; $\left.\mathrm{AD}, R^{2}=0.13\right)$, but rather appeared to be a more constant "state" of high-frequency activity maintained throughout the experiment. The unique benefit of ICA is that it can identify temporal patterns of activity that are not merely varying with the task paradigm. It is important to note that the positive and negative activity for this component are reported not as a function of the task. Instead this component showed evidence of sustained positive activity above the baseline mean signal over the entire experimental run. Therefore, the positive activity within the $\mathrm{Sec}-\mathrm{C}$ does not represent "activation" as commonly described in relation to being locked to the paradigm timing.

The spatial pattern of the Sec-C overlapped many of the regions that showed evidence of decreased activity in the Neg-TRC, particularly posteriorly, in lateral and medial parietal regions. The spatial pattern of Sec-C activity in the parietal regions and posterior cingulate was remarkably consistent across all groups, but interestingly, the NC and low-SB MCI groups also showed anterior cingulate and dorsolateral prefrontal activity (NC: $Z=3.9, x=-51, y=30, z=27, k=138$, and $Z=3.3, x=36$, $y=30, z=42, k=286$; low-SB MCI: $Z=5.4, x=-45, y=27$, $z=96, k=317$, and $Z=5.6, x=42, y=36 ; z=30, k=494$ ) (Fig. 7). This combination of prefrontal and parietal regions was similar to networks previously implicated in attention and cognitive control (Gabrieli, 1996; Miller, 2000; Milham et al., 2002).

\section{Multiple regression: nonlinear model within the secondary component of interest}

The multiple regression model testing for a nonlinear trajectory over the course of MCI was applied to Sec-C to confirm the between group findings, as well as determine whether the network represented by this component also disrupted in a similar pattern to the task-related activity. The regions displaying the strongest positive relationship to the model include lateral aspects of the inferior frontal gyrus, posterior cingulate, and the dorsal anterior cingulate (Table 2). Interestingly, posterior parietal regions, including both the precuneus and superior lateral 


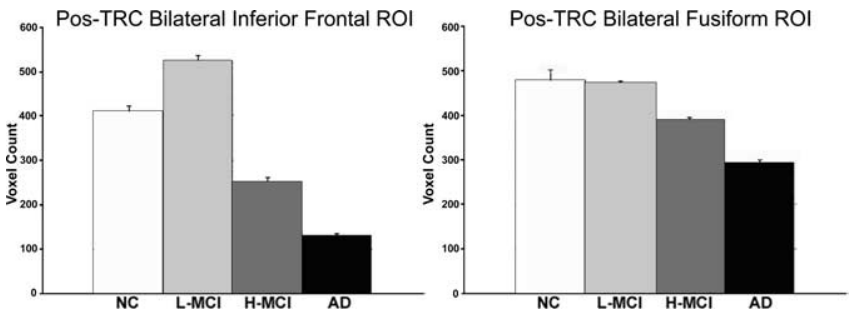

Figure 6. Pos-TRC ROI analysis. Bar graphs display the results from the ROI voxel count analyses (+SE). $\boldsymbol{a}$, Low-SB MCl (L-MCl) subjects demonstrated hyperactivation within the bilateral inferior frontal ROI, supporting the theory of a nonlinear trajectory over the course of preclinical AD. All groups were significantly different $(p<0.0001)$. $\boldsymbol{b}$, The bilateral fusiform $\mathrm{RO}$ I did not appear to be a region sensitive to hyperactivation. All between-group differences were significant within both ROls ( $p<0.0001$ ), except for the difference between NC and Iow-SB MCI subjects within the fusiform ROI ( $p=0.73)$.

parietal regions demonstrated activity in the inverse pattern to the proposed regression model $(\mathrm{AD}>$ high-SB MCI $>$ normal $>$ low-SB MCI) suggesting an increased level of positive activity in these regions observed in the high-SB MCI and AD subjects (see below, Secondary component of interest, between-group analysis).

\section{Secondary component of interest, between-group analysis} The between-group analyses of the Sec-C confirmed that all groups displayed a similar spatial parietal pattern of activation, but somewhat surprisingly, the high-SB MCI and AD groups exhibited a significantly greater expression of the Sec-C within the superior lateral parietal regions compared with $\mathrm{NC}$ and low-SB MCI (high-SB MCI > NC: $Z=5.1, x=-30, y=-57$, $z=66, k=241$, and $Z=5.4, x=27, y=-54, z=69, k=144$; $\mathrm{AD}>\mathrm{NC}: Z=5.4, x=-30, y=-42, z=66, k=163$, and $Z=$ 4.7, $x=39, y=-45, z=60, k=7)$. These regions showing increased positive Sec-C activity overlapped the areas showing decreased expression of the Neg-TRC, and are thought to be an integral part of the "default network" that is altered in MCI and $\mathrm{AD}$. The $\mathrm{AD}$ group demonstrated significantly less expression of the $\mathrm{Sec}-\mathrm{C}$ in dorsolateral prefrontal and medial frontal cortices, as well as the anterior cingulate, compared with the low-SB MCI, high-SB MCI, and NC groups $(p<0.0001)$.

\section{Relationship between Neg-TRC and secondary component of interest}

To further examine the overlap in regions between the Sec-C and the Neg-TRC, we explored the relationship of the extent of voxels in each of these components within a specific ROI. Lateral parietal regions showed an indirect correlation between the Neg-TRC activity and the Sec-C activity $(r=-0.552 ; p<0.0001)$. That is, greater expression of positive activity within the $\mathrm{Sec}-\mathrm{C}$ was associated with less deactivation in the task-related component. Also, the extent of Neg-TRC activity within the superior frontal gryus and the anterior cingulate indirectly correlated with the amount of superior frontal gyrus in the Sec-C $(r=-0.292, p<0.04 ; r=$ $-833, p<0.0001$, respectively).

\section{Postscan memory test performance}

The NC, low-SB MCI, and high-SB MCI groups all performed relatively well on both postscan memory tests (face recognition: NC, $74.8 \pm 14.6 \%$; low-SB MCI, $78.8 \pm 10.1 \%$; high-SB MCI, $75.3 \pm 10.8 \%$; forced-choice name recognition: NC, $87.7 \pm$ 10.7\%; low-SB MCI, $83.0 \pm$ 9.0\%; high-SB MCI, $87.0 \pm 13.3 \%$ ). The AD subjects performed above chance levels, but significantly worse than the other groups on both memory tests (face recognition: $\mathrm{AD}, 64.6 \pm 10.6 \%$; forced-choice name recognition: $\mathrm{AD}$, $65.7 \pm 11.8 \%, p<0.05)$ than the NC and both low-SB and high-SB MCI subjects. Performance on these tests was not correlated with subject's age, but was correlated to the degree of cognitive impairment, as assessed generally by the MMSE (face recognition: $r=0.299, p<0.03$; forced-choice name recognition: $r=0.522, p<0.0001)$.

\section{Relationship of component activity and postscan memory test scores}

The percentage of correct responses from MT1 (face recognition) and MT2 (forced-choice name recognition) were entered into two separate correlations across group to measure the relationship of activity within the component and memory performance. Correct responses on MT1 consisted of correct identification of faces viewed only once during scanning. MT2 required the correct identification of the name that was paired with the faces viewed only once during scanning. The correlation of performance on both memory tests to the Pos-TRC was conducted and regions that demonstrated a strong correlation at the $p<0.01$ level were further investigated. The Pos-TRC activity displays a correlation between the hippocampus with MT1 $(Z=2.6$; $p<$ $0.005 ; x=-27, y=-18, z=-12)$, and a weaker, but still significant correlation within the hippocampus for MT2 $(Z=$ 2.3; $p<0.01 ; x=27, y=-18, z=-6)$. Within the Neg-TRC, significant direct correlations were observed along the cingulate gyrus (MT1: $Z=2.8, p<0.003, x=-6, y=-18, z=-36$; MT2: $Z=3.1, p<0.001, x=-9, y=-18, z=39)$ as well as medial parietal regions (MT1: $Z=2.3, p<0.01, x=-12, y=-45, z=$ 39; MT2: $Z=3.2, p<0.001, x=-18, y=45, z=33$ ) for both memory tests. This directionality of this correlation may be somewhat counterintuitive, but the direct correlation in this case represents evidence of greater deactivation as memory performance increases. Finally, the results from the Sec-C highlight the direct relationship between the "state activity" in medial and dorsolateral prefrontal regions [MT1: medial frontal $(Z=$ $2.5 ; p<0.003 ; x=0, y=33, z=42)$, dorsolateral prefrontal $(Z=3.4 ; x=39, y=36, z=33$; and $Z=3.2 ; p<0.001 ; x=$ $-51, y=12, z=24)$; MT2: medial frontal $(Z=2.69 ; p<$ $0.004 ; x=3, y=18, z=48)$, dorsolateral prefrontal $(Z=2.39$; $p<0.008 ; x=-33, y=36, z=36$; and $Z=3.9 ; x=36, y=$ $27, z=33)$ ] and memory performance on both memory tests.

\section{Discussion}

In this study, we used ICA to examine patterns of memoryrelated neural activity across the continuum of normal aging to mild AD. We found a pattern of brain regions demonstrating temporally synchronous fMRI activity, supporting the hypothesis that a specific set of large-scale distributed brain networks mediates the process of associative encoding. Of particular interest was the evidence for a strong reciprocal relationship between the degree of memory-related activation within the hippocampus and deactivation of medial and lateral parietal regions. Furthermore, our results provide support for a nonlinear trajectory of memory-related activation and deactivation over the course of prodromal AD. Subjects at the very mild end of the cognitive impairment continuum demonstrated evidence of paradoxically increased activation not only in the hippocampus and functionally connected neocortical regions, but also increased deactivation in the default network compared with controls. Subjects at the more impaired end of the MCI continuum showed significantly decreased hippocampal activation and reduced deactiva- 


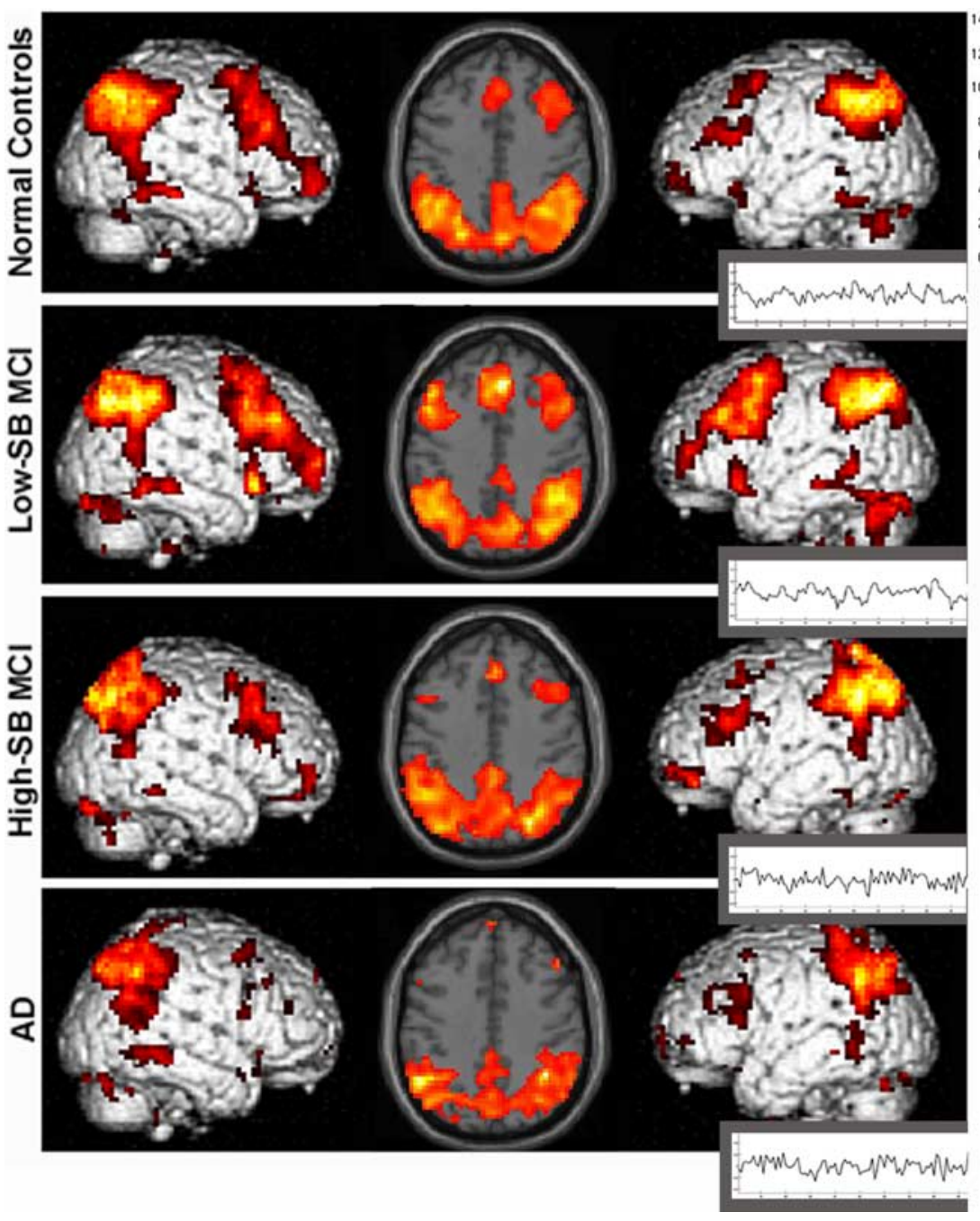

Figure 7. Within-group Sec-C. Axial slices display positive activity within the $\operatorname{Sec}-\mathrm{C}(p<0.01)$. The left hemisphere is displayed on the left. The colorscale represents $\mathrm{T}$ scores of the significance of each voxel contributing to the overall temporal component. It is important to note that although this is positive activity relative to the temporal pattern of $\mathrm{Sec}-\mathrm{C}$, this component does not represent activation as commonly described in relation to task-related activity, as there is little evidence of signal change that is time-linked to the paradigm. Although this component was not clearly task related in terms of relationship to the paradigm timing, there was evidence of sustained positive activity that was spatially consistent across the normal control, low-SB MCl, and high-SB MCI groups, however the AD patients lack the lateral and medial frontal positive activity seen in the other groups.

the hippocampus is functionally connected to medial and lateral and medial frontal regions and may serve as a memory modulator (Greicius et al., 2003). Our findings suggest that the recent reports of alterations in default network activity in MCI and AD patients (Lustig et al., 2003; Greicius et al., 2004; Rombouts et al., 2005a) are related to alterations in the functional integrity of the hippocampalbased memory system. It may be that the successful engagement of the MTL in a memory task, particularly during the encoding of novel information, may require concurrent disengagement of the default network (Daselaar et al., 2004). Alternatively, activation of the MTL may suppress activity within the default network. Other groups have also suggested that these parietal regions may be involved in successful memory retrieval, and have also been characterized as a "retrosplenial memory system” (Buckner et al., 2005). Interestingly, our recent fMRI studies in young subjects have suggested that these parietal regions may be specifically involved in metamemory processes, in particular the assessment of one's own memory performance (Chua et al., 2006).

We also found evidence of a neocortical component with a very different temporal pattern of activity (Sec-C). This secondary component involved a set of parietal regions, which includes posterior cingulate and parietal regions, but also dorsolateral prefrontal and anterior cingulate regions. A similar neocortical network has been described in tasks requiring sustained attentional or cognitive control (Gabrieli, 1996; Miller, 2000; Milham et al., 2002). This attentional system likely also impacts the process of associative encoding, perhaps representing a more sustained attentional state, or "behavioral set," as greater ex-

tion in default regions, in a pattern similar to patients diagnosed with mild AD. Finally, we found the pattern of task-related deactivation was associated with altered expression of more tonic or state activity within a neocortical network thought to subserve attentional processes. These results strongly suggest that widely distributed memory networks are altered over the course of prodromal $\mathrm{AD}$, and provide evidence of a functional interaction between medial temporal lobe and neocortical pathology in early AD.

To our knowledge, these results provide the first direct evidence that activation within the hippocampus during an episodic memory paradigm is specifically related to deactivation of regions that have been characterized previously as the "default mode network" (Raichle et al., 2001). Previous work has suggested that the MTL and these parietal regions may be directly connected. Animal studies have demonstrated that lesioning the MTL deafferents lateral and medial parietal regions (Meguro et al., 2001). Previous fMRI studies of "resting state" connectivity have also suggested that even when not engaged in a specific task, pression in cingulate and prefrontal regions correlated with better postscan memory performance. Alterations in this neocortical attentional network might also be related to deficits in executive function frequently observed in MCI and AD (Albert et al., 2001). Furthermore, loss of task-related deactivation within the default network was related to increased expression of positive activity in these same parietal regions, consistent with the theory that loss of ability to regulate or functionally inhibit activation may underlie the failure of deactivation or default mode activity in MCI and AD (Lustig et al., 2003).

Our findings also provide additional support for the hypothesis of a nonlinear trajectory of fMRI activation over the course of prodromal AD. Our previous studies using anatomically defined ROI methods have demonstrated paradoxically increased activation in posterior hippocampal and parahippocampal regions during a scene encoding task in a separate group of MCI subjects (Dickerson et al., 2004), and in the anterior hippocampus and entorhinal cortex using this face-name paradigm in a subset of the subjects reported in the current study (Dickerson et al., 2005). 
In addition, a number of fMRI studies in subjects with genetic risk factors for AD (Bookheimer et al., 2000; Smith et al., 2002; Bondi et al., 2005) or strong family history (Bassett et al., 2006) have found evidence of increased activation before significant cognitive decline. This phenomena may represent a compensatory process (i.e., increased neuronal recruitment) in the setting of early pathology, associated biochemical alterations (i.e., upregulation of choline acetyltransferase activity) (DeKosky et al., 2002), a direct result of the pathophysiological process of AD (i.e., aberrant axonal sprouting) (Masliah et al., 2003), or disruption of synaptic integration (Stern et al., 2004). It is also possible this hyperactivation is the result of additional conscious effort or affective valence in very mildly impaired subjects who may be particularly concerned about their memory performance.

Importantly, these results also provide some explanation for discrepant results among previously published fMRI studies in $\mathrm{MCI}$, as this is one of the first studies to characterize a priori the heterogenous group of individuals termed "MCI" along the group's continuum of impairment. The more impaired MCI subjects (based on CDR-SB score), showed a pattern of decreased activation in the MTL and functionally connected regions that strongly resembled the pattern seen in mild AD subjects. These results are similar to reports from other groups who have studied more impaired MCI subjects (Machulda et al., 2003; Johnson et al., 2006), and suggests that the variability in published fMRI studies of MCI is likely related to the heterogeneity of subjects within the MCI continuum. Furthermore, our findings are consistent with the theory that the late stage of MCI is likely already very mild AD (Morris and Cummings, 2005). Longitudinal follow-up is necessary, however, to determine whether the MCI subjects in the present study are indeed in the early phases of $\mathrm{AD}$. Previous work by our group suggests that many such subjects "convert" to clinical AD over 3 years (Daly et al., 2000). However, because we did not require specific cutoff scores on neuropsychological tests to demonstrate objective memory impairment, even the high-SB MCI subjects in this study may not be fully comparable with those in other studies. In addition, our subjects are highly educated, and the issue of "cognitive reserve" remains to be explored.

Several additional factors need to be considered in the observed findings. Statistical power was of potential concern given the smaller sample of $\mathrm{AD}$ patients compared with the other groups; however, evidence of greater activity in some regions within the Neg-TRC in the AD group suggests adequate power to detect group differences in both directions. The data were sampled within standardized space and, thus, vulnerable to the confounding effects of atrophy. Our previous studies in MCI and AD using anatomically based ROI, however, have yielded similar results in the pattern of hippocampal activation even accounting for the contribution of volume. Ultimately, the combination of analyses using individual subject anatomically defined ROIs and whole-brain multivariate analyses to examine large scale networks should prove particularly valuable in understanding the evolution of both structural and functional alterations of the course of MCI and AD.

These results may have interesting implications for the interaction of neocortical and MTL-based pathology in AD. Importantly, as discussed by Buckner et al. (2005), AD patients demonstrate significant reductions in resting cerebral glucose metabolism and perfusion on positron emission tomography (PET) and single photon emission computed tomography studies in a strikingly similar default network of medial and lateral parietal regions that typically deactivate in $\mathrm{AMRI}$ and PET para- digms. This anatomical distribution also overlaps the pattern of neocortical retention of the PET ligand Pittsburgh Compound B (PIB) labeling fibrillar amyloid (Klunk et al., 2004; Buckner et al., 2005). Interestingly, PIB retention is only modestly elevated in the MTL of AD patients (Klunk et al., 2004), suggesting that the amyloid may exert its effects more remotely, perhaps from the end-terminal fields of hippocampal projections to these parietal regions. Volumetric imaging studies with postmortem correlation have suggested that tau-based pathology (i.e., neurofibrillary tangles) is more strongly associated with hippocampal atrophy than with amyloid levels (Csernansky et al., 2004), and our findings may simply reflect the fact that amyloid and tau pathology co-occur. Future studies combining PIB amyloid imaging with structural and functional MRI studies of memory processes should shed light on the association of neocortical amyloid deposition to hippocampal dysfunction, as our findings suggest a strong reciprocal relationship between alterations in neural activity in these memory networks over the course of prodromal AD.

\section{References}

Albert MS, Moss MB, Tanzi R, Jones K (2001) Preclinical prediction of AD using neuropsychological tests. J Int Neuropsychol Soc 7:631-639.

Bassett SS, Yousem DM, Cristinzio C, Kusevic I, Yassa MA, Caffo BS, Zeger SL (2006) Familial risk for Alzheimer's disease alters fMRI activation patterns. Brain 129:1229-1239.

Bell AJ, Sejnowski TJ (1995) An information-maximization approach to blind separation and blind deconvolution. Neural Comput 7:1129-1159.

Bondi MW, Houston WS, Eyler LT, Brown GG (2005) fMRI evidence of compensatory mechanisms in older adults at genetic risk for Alzheimer disease. Neurology 64:501-508.

Bookheimer SY, Strojwas MH, Cohen MS, Saunders AM, Pericak-Vance MA, Mazziotta JC, Small GW (2000) Patterns of brain activation in people at risk for Alzheimer's disease. N Engl J Med 343:450-456.

Brett M, Johnsrude IS, Owen AM (2002) The problem of functional localization in the human brain. Nat Rev Neurosci 3:243-249.

Buckner RL, Snyder AZ, Shannon BJ, LaRossa G, Sachs R, Fotenos AF, Sheline YI, Klunk WE, Mathis CA, Morris JC, Mintun MA (2005) Molecular, structural, and functional characterization of Alzheimer's disease: evidence for a relationship between default activity, amyloid, and memory. J Neurosci 25:7709-7717.

Calhoun VD, Adali T, Pearlson GD, Pekar JJ (2001) A method for making group inferences from functional MRI data using independent component analysis. Hum Brain Mapp 14:140-151.

Chua EF, Schacter DL, Rand-Giovannetti E, Sperling RA (2006) Understanding metamemory: neural correlates of the cognitive process and subjective level of confidence in recognition memory. NeuroImage 29:1150-1160.

Correa N, Adali T, Li Y, Calhoun V (2005) Comparison of blind source separation algorithms for fMRI using a new matlab toolbox: GIFT. Proc IEEE Int Conf Acoustics, Speech, Signal Processing 5:401-404.

Csernansky JG, Hamstra J, Wang L, McKeel D, Price JL, Gado M, Morris JC (2004) Correlations between antemortem hippocampal volume and postmortem neuropathology in AD subjects. Alzheimer Dis Assoc Disord 18:190-195.

Daly E, Zaitchik D, Copeland M, Schmahmann J, Gunther J, Albert M (2000) Predicting conversion to Alzheimer disease using standardized clinical information. Arch Neurol 57:675-680.

Daselaar SM, Prince SE, Cabeza R (2004) When less means more: deactivations during encoding that predict subsequent memory. NeuroImage 23:921-927.

DeKosky ST, Ikonomovic MD, Styren SD, Beckett L, Wisniewski S, Bennett DA, Cochran EJ, Kordower JH, Mufson EJ (2002) Upregulation of choline acetyltransferase activity in hippocampus and frontal cortex of elderly subjects with mild cognitive impairment. Ann Neurol 51:145-155.

Dickerson BC, Salat DH, Bates JF, Atiya M, Killiany RJ, Greve DN, Dale AM, Stern CE, Blacker D, Albert MS, Sperling RA (2004) Medial temporal lobe function and structure in mild cognitive impairment. Ann Neurol $56: 27-35$. 
Dickerson BC, Salat DH, Greve DN, Chua EF, Rand-Giovannetti E, Rentz DM, Bertram L, Mullin K, Tanzi RE, Blacker D, Albert MS, Sperling RA (2005) Increased hippocampal activation in mild cognitive impairment compared to normal aging and AD. Neurology 65:404-411.

Gabrieli JD (1996) Memory systems analyses of mnemonic disorders in aging and age-related diseases. Proc Natl Acad Sci USA 93:13534-13540.

Greicius MD, Krasnow B, Reiss AL, Menon V (2003) Functional connectivity in the resting brain: a network analysis of the default mode hypothesis. Proc Natl Acad Sci USA 100:253-258.

Greicius MD, Srivastava G, Reiss AL, Menon V (2004) Default-mode network activity distinguishes Alzheimer's disease from healthy aging: evidence from functional MRI. Proc Natl Acad Sci USA 101:4637-4642.

Hyman BT, Van Hoesen GW, Damasio AR, Barnes CL (1984) Alzheimer's disease: cell-specific pathology isolates the hippocampal formation. Science 225:1168-1170.

Johnson SC, Baxter LC, Susskind-Wilder L, Connor DJ, Sabbagh MN, Caselli RJ (2004) Hippocampal adaptation to face repetition in healthy elderly and mild cognitive impairment. Neuropsychologia 42:980-989.

Johnson SC, Schmitz TW, Moritz CH, Meyerand ME, Rowley HA, Alexander AL, Hansen KW, Gleason CE, Carlsson CM, Ries ML, Asthana S, Chen K, Reiman EM, Alexander GE (2006) Activation of brain regions vulnerable to Alzheimer's disease: The effect of mild cognitive impairment. Neurobiol Aging, in press.

Klunk WE, Engler H, Nordberg A, Wang Y, Blomqvist G, Holt DP, Bergstrom M, Savitcheva I, Huang GF, Estrada S, Ausen B, Debnath ML, Barletta J, Price JC, Sandell J, Lopresti BJ, Wall A, Koivisto P, Antoni G, Mathis CA, et al. (2004) Imaging brain amyloid in Alzheimer's disease with Pittsburgh Compound-B. Ann Neurol 55:306-319.

Lustig C, Snyder AZ, Bhakta M, O’Brien KC, McAvoy M, Raichle ME, Morris JC, Buckner RL (2003) Functional deactivations: change with age and dementia of the Alzheimer type. Proc Natl Acad Sci USA 100:14504-14509.

Machulda MM, Ward HA, Borowski B, Gunter JL, Cha RH, O’Brien PC, Petersen RC, Boeve BF, Knopman D, Tang-Wai DF, Ivnik RJ, Smith GE, Tangalos EG, Jack Jr CR (2003) Comparison of memory fMRI response among normal, MCI, and Alzheimer's patients. Neurology 61:500-506.

Marchini JL, Ripley BD (2000) A new statistical approach to detecting significant activation in functional MRI. NeuroImage 12:366-380.

Masliah E, Alford M, Adame A, Rockenstein E, Galasko D, Salmon D, Hansen LA, Thal LJ (2003) Abetal-42 promotes cholinergic sprouting in patients with AD and Lewy body variant of AD. Neurology 61:206-211.

McKhann G, Drachman D, Folstein M, Katzman R, Price D, Stadlan EM (1984) Clinical diagnosis of Alzheimer's disease: report of the NINCDSADRDA Work Group under the auspices of Department of Health and Human Services Task Force on Alzheimer's Disease. Neurology 34:939-944

Meguro K, LeMestric C, Landeau B, Desgranges B, Eustache F, Baron JC (2001) Relations between hypometabolism in the posterior association neocortex and hippocampal atrophy in Alzheimer's disease: a PET/MRI correlative study. J Neurol Neurosurg Psychiatry 71:315-321.

Milham MP, Erickson KI, Banich MT, Kramer AF, Webb A, Wszalek T, Cohen NJ (2002) Attentional control in the aging brain: insights from an fMRI study of the stroop task. Brain Cogn 49:277-296.
Miller EK (2000) The prefrontal cortex and cognitive control. Nat Rev Neurosci 1:59-65.

Moritz CH, Rogers BP, Meyerand ME (2003) Power spectrum ranked independent component analysis of a periodic fMRI complex motor paradigm. Hum Brain Mapp 18:111-122.

Morris JC, Cummings J (2005) Mild cognitive impairment (MCI) represents early-stage Alzheimer's disease. J Alzheimers Dis 7:235-262.

Morris JC, Ernesto C, Schafer K, Coats M, Leon S, Sano M, Thal LJ, Woodbury $\mathrm{P}$ (1997) Clinical dementia rating training and reliability in multicenter studies: the Alzheimer's disease cooperative study experience. Neurology 48:1508-1510.

Petersen RC, Smith GE, Waring SC, Ivnik RJ, Kokmen E, Tangelos EG (1997) Aging, memory, and mild cognitive impairment. Int Psychogeriatr 9:65-69.

Raichle ME, MacLeod AM, Snyder AZ, Powers WJ, Gusnard DA, Shulman GL (2001) A default mode of brain function. Proc Natl Acad Sci USA 98:676-682.

Rombouts SA, Goekoop R, Stam CJ, Barkhof F, Scheltens P (2005a) Delayed rather than decreased BOLD response as a marker for early Alzheimer's disease. NeuroImage 26:1078-1085.

Rombouts SA, Barkhof F, Goekoop R, Stam CJ, Scheltens P (2005b) Altered resting state networks in mild cognitive impairment and mild Alzheimer's disease: an fMRI study. Hum Brain Mapp 26:231-239.

Schmithorst VJ, Holland SK (2004) Comparison of three methods for generating group statistical inferences from independent component analysis of functional magnetic resonance imaging data. J Magn Reson Imaging 19:365-368.

Small SA, Perera GM, DeLaPaz R, Mayeux R, Stern Y (1999) Differential regional dysfunction of the hippocampal formation among elderly with memory decline and Alzheimer's disease. Ann Neurol 45:466-472.

Smith CD, Andersen AH, Kryscio RJ, Schmitt FA, Kindy MS, Blonder LX, Avison MJ (2002) Women at risk for AD show increased parietal activation during a fluency task. Neurology 58:1197-1202.

Sperling R, Bates J, Chua E, Cocchiarella A, Schacter DL, Rosen B, Albert M (2003a) fMRI studies of associative encoding in young and elderly controls and mild AD patients. J Neurol Neurosurg Psychiatry 74:44-50.

Sperling R, Chua E, Cocchiarella A, Rand-Giovannetti E, Poldrack R, Schacter DL, Albert M (2003b) Putting names to faces: successful encoding of associative memories activates the anterior hippocampal formation. NeuroImage 20:1400-1410.

Sperling RA, Bates J, Cocchiarella A, Schacter D, Rosen B, Albert M (2001) Encoding novel face-name associations: a functional MRI study. Hum Brain Mapp 14:129-139.

Sperling RA, Greve D, Dale A, Killiany R, Rosen B, Holmes J, Rosas HD, Cocchiarella A, Firth P, Lake S, Lange N, Routledge C, Albert M (2002) fMRI detection of pharmacologically induced memory impairment. Proc Natl Acad Sci USA 99:455-460.

Stern EA, Bacskai BJ, Hickey GA, Attenello FJ, Lombardo JA, Hyman BT (2004) Cortical synaptic integration in vivo is disrupted by amyloid- $\beta$ plaques. J Neurosci 24:4535-4540. 